\title{
Diagnostic value of [18F]FDG-PET/CT in polymyalgia rheumatica: a systematic review and meta-analysis
}

\author{
K. S. M. van der Geest ${ }^{1}$ - G. Treglia ${ }^{2,3,4,5}$ - A. W. J. M. Glaudemans ${ }^{6}$ - E. Brouwer ${ }^{1}$ • F. Jamar ${ }^{7}$. \\ R. H. J. A. Slart ${ }^{6,8} \cdot$ O. Gheysens ${ }^{7}$
}

Received: 16 October 2020 / Accepted: 8 December 2020 / Published online: 28 December 2020

(C) The Author(s) 2020

\begin{abstract}
Purpose Polymyalgia rheumatica (PMR) can be difficult to diagnose. Whole-body [18F]FDG-PET/CT allows for a comprehensive evaluation of all relevant articular and extra-articular structures affected by PMR. We aimed to summarize current evidence on the diagnostic value of [18F]FDG-PET/CT for a diagnosis of PMR.

Methods PubMed/MEDLINE and the Cochrane Library database were searched from inception through May 31, 2020. Studies containing patients with PMR who underwent [18F]FDG-PET/CT were included. Screening and full-text review were performed by 3 investigators and data extraction by 2 investigators. Risk of bias was examined with the QUADAS-2 tool. Diagnostic test meta-analysis was performed with a bivariate model.

Results Twenty studies were included in the systematic review, of which 9 studies ( $n=636$ patients) were eligible for metaanalysis. [18F]FDG positivity at the following sites was associated with a diagnosis of PMR: interspinous bursae (positive likelihood ratio (LR+) 4.00; 95\% CI 1.84-8.71), hips (LR+ 2.91; 95\% CI 2.09-4.05), ischial tuberosities (LR+ 2.86; 95\% CI 1.91-4.28), shoulders (LR+ 2.57; 95\% CI 1.24-5.32) and sternoclavicular joints (LR+ 2.31; 95\% CI 1.33-4.02). Negative likelihood ratios (LR-) for these sites, as well as the greater trochanters, were all less than 0.50. Composite [18F]FDG-PET/ CT scores, as reported in 3 studies, provided a pooled LR+ of 3.91 (95\% CI 2.42-6.32) and LR- of 0.19 (95\% CI 0.10-0.36). Moderate to high heterogeneity was observed across the studies, mainly due to differences in patient selection, scanning procedures and/or interpretation criteria.

Conclusion Significant [18F]FDG uptake at a combination of anatomic sites is informative for a diagnosis of PMR. [18F]FDG$\mathrm{PET} / \mathrm{CT}$ might be an important diagnostic tool in patients with suspected PMR. This study also highlights the need for adherence to published procedural recommendations and standardized interpretation criteria for the use of [18F]FDG-PET/CT in PMR.
\end{abstract}

Keywords Polymyalgia rheumatica · Positron emission tomography/computed tomography · Fluorodeoxyglucose F18 . Meta-analysis · Review

This article is part of the Topical Collection on Infection and inflammation

K. S. M. van der Geest

k.s.m.van.der.geest@umcg.nl

1 Department of Rheumatology and Clinical Immunology, University of Groningen, University Medical Center Groningen, Hanzeplein 1, 9700RB Groningen, the Netherlands

2 Clinic of Nuclear Medicine and PET/CT Center, Imaging Institute of Southern Switzerland, Ente Ospedaliero Cantonale, Bellinzona, Switzerland

3 Clinic of Nuclear Medicine and PET/CT Center, Imaging Institute of Southern Switzerland, Ente Ospedaliero Cantonale, Lugano, Switzerland
4 Department of Nuclear Medicine and Molecular Imaging, Lausanne University Hospital, University of Lausanne, Lausanne, Switzerland

5 Health Technology Assessment Unit, Academic Education, Research and Innovation Area, Ente Ospedaliero Cantonale, Bellinzona, Switzerland

6 Department of Nuclear Medicine and Molecular Imaging, University of Groningen, University Medical Center Groningen, Groningen, The Netherlands

7 Department of Nuclear Medicine, Cliniques Universitaires Saint-Luc and Institute of Clinical and Experimental Research (IREC), Université Catholique de Louvain (UCLouvain), Brussels, Belgium

8 Department of Biomedical Photonic Imaging, Faculty of Science and Technology, University of Twente, Enschede, The Netherlands 


\section{Introduction}

Polymyalgia rheumatica (PMR) is the most common rheumatic inflammatory disease above the age of 50. It is characterized by inflammation of articular and peri-articular structures causing debilitating pain and stiffness of the shoulders and hips [1, 2]. PMR is associated with large vessel inflammation, i.e. giant cell arteritis, in approximately $20 \%$ of patients [2]. Inflammatory markers, such as the erythrocyte sedimentation rate (ESR) and C-reactive protein (CRP) level, are usually elevated in patients with PMR [3]. Several classification criteria have been proposed for PMR but these are not intended for diagnostic use [2]. There are no disease-specific symptoms or laboratory markers for PMR. The discrimination between PMR and its mimicking conditions can be very challenging. Since the treatment differs, the presence of other rheumatic diseases (e.g. late-onset rheumatoid arthritis, lateonset spondyloarthritis, osteoarthritis) as well as parainfectious myalgia and neoplastic diseases should be ruled out [4].

Various imaging modalities have been introduced in the diagnostic work-up of suspected PMR. Ultrasonography and magnetic resonance imaging (MRI) may reveal subacromialsubdeltoid bursitis, biceps tenosynovitis, glenohumeral synovitis, coxofemoral synovitis and/or trochanteric bursitis [2, 5-9]. These abnormalities are more accurately detected by MRI than ultrasonography [10]. MRI scans covering selected areas (e.g. shoulder and hip girdle) and also total body MRI may be helpful in the evaluation of PMR [7-10].

An emerging imaging tool for PMR might be 2-deoxy2-[18F]fluoro-D-glucose ([18F]FDG) positron emission tomography combined with low-dose computed tomography ([18F]FDG-PET/CT). This imaging modality is wellestablished in oncology and has an expanding role in the assessment of inflammatory conditions [11, 12]. [18F]FDG enters activated immune cells and fibroblasts through the glucose transporter [13, 14]. Importantly, [18F]FDG-PET/CT allows for a comprehensive evaluation of all relevant articular and extra-articular structures in a patient with suspected PMR and may aid in the differentiation between PMR and other rheumatic inflammatory conditions $[12,15]$. Furthermore, [18F]FDG$\mathrm{PET} / \mathrm{CT}$ allows ruling out concomitant large vessel vasculitis and other serious conditions [16]. In the current systematic review and meta-analysis, we aimed to summarize the growing evidence on the diagnostic value of [18F]FDG-PET/CT for a diagnosis of PMR.

\section{Methods}

A predefined study protocol was established but not registered. This study is reported in agreement with the Preferred Reporting Items for a Systematic Review and Meta-Analysis
(PRISMA) statement [17]. No ethical approval or informed consent was required.

\section{Search strategy}

A comprehensive search of records through the PubMed/ MEDLINE and Cochrane Library databases was carried out (date of last search: May 31, 2020). The following search algorithm was used: (A) 'PET' OR 'positron emission tomography' OR 'FDG' OR 'fluorodeoxyglucose' AND (B) 'PMR' OR 'polymyalgia'. There were neither date limits nor language restrictions applied to the database search. In order to achieve a more comprehensive search, the references of the selected articles were screened manually.

\section{Study selection}

Titles and abstracts of the records were independently screened by three reviewers (OG, GT and KSMG). Studies were selected for the systematic review according to predefined criteria. Inclusion criteria were original articles reporting [18F]FDG-PET/CT findings in patients with PMR. The reference standard for PMR could be classification criteria or a clinical diagnosis made by the treating physician. Exclusion criteria were as follows: (a) reviews, editorials, comments, study protocols; (b) case reports (less than 5 patients included); (c) articles outside the field of interest of this review (e.g. articles focused on [18F]FDG-PET without CT, articles including patients with giant cell arteritis rather than PMR); (d) articles not available in English. Subsequently, studies providing sufficient data on the diagnostic accuracy of [18F]FDG-PET/CT (i.e. the index text) for a diagnosis of PMR were included in the meta-analysis. Potential overlap of patients between studies from the same hospital was evaluated for studies in the meta-analysis. In case of possible overlap in patients, data was obtained from one study only and priority was given according to criteria in the following order: (1) a study with patients who were not (yet) treated with glucocorticoids, (2) a study with the largest number of patients, (3) a study reporting a clear definition of PET positivity, (4) a study including control subjects who were suspected of having PMR and (5) a study including control subjects with rheumatoid arthritis or another rheumatic inflammatory disease. Disagreements were solved through an online consensus meeting between the reviewers.

\section{Data extraction}

Two reviewers (OG, GT) independently collected information about study characteristics (i.e. authors, year of publication, country, study design) and patient characteristics (i.e. patient population, criteria used for PMR diagnosis, age, sex ratio, 
number of PMR patients evaluated and [18F]FDG-PET/CT scans performed, immunosuppressive treatment, presence of a control group). Two independent reviewers (KSMG, RS) also collected data on technical details (i.e. [18F]FDGPET/CT imaging modality, [18F]FDG injected activity, time interval between [18F]FDG injection and image acquisition, scan coverage, [18F]FDG-PET/CT image analysis and definition of positive findings) and any data on the per-patient diagnostic accuracy of [18F]FDG-PET/CT for PMR (i.e. true positive and true negative findings, false positive and false negative findings). Authors of studies were not contacted.

\section{Quality assessment}

The quality of the studies included in the meta-analysis was assessed according to the revised 'Quality Assessment of Diagnostic Accuracy Studies' tool (QUADAS-2) [18]. The latter was used to assess the risk of bias for the following criteria: patient selection, index test, reference test and flow/timing whereas applicability concerns were assessed for patient selection, index test and reference test.

\section{Statistical analysis}

A bivariate model analysis was performed to assess the summary estimates of sensitivity, specificity, diagnostic odds ratio (DOR), positive likelihood ratio (LR+) and negative likelihood ratio (LR-). Pooled data were given with $95 \%$ confidence intervals $(95 \% \mathrm{CI})$ and displayed using forest plots and hierarchical summary receiver operating characteristics (HSROC) plots. Likelihood ratios of more than 2.00 or less than 0.50 with $95 \% \mathrm{CI}$ not including 1.00 were considered statistically significant. The bivariate model analysis could not be used for findings reported by less than four studies. In that case, pooled estimates of the diagnostic parameters were determined with a univariate random effects model (DerSimonian-Laird method) and summary estimates were only shown if heterogeneity $\left(I^{2}\right)$ was $<75 \%$. Bivariate model analysis and HSROC plots were performed with STATA version 15.1 (metandi command). Univariate models were evaluated with MetaDiSc version 1.4 and forest plots were constructed in Review Manager version 5.3. No sub-analyses were performed.

\section{Results}

\section{Literature search}

A total of 231 records were identified through the comprehensive electronic database search (Fig. 1), with the oldest reference dating from May 1999 [19]. Two hundred ten records were excluded after title/abstract screening and 1 record after full-text evaluation [20]. Thus, 20 articles ( $n=694$ patients with PMR) were included in the qualitative analysis (systematic review) [21-40]. Subsequently, 11 of these studies were excluded from the meta-analysis due to lack of a control group ( 8 studies), inclusion of patients with giant cell arteritis without PMR (1 study), part of patients undergoing [18F]FDG-PET without CT (1 study) and one study reporting on muscle metabolic activity in patients with PMR rather than [18F]FDG uptake in typical joints, bursae and/or tendon entheses. Ultimately, 9 studies containing 636 patients (of which 253 patients had PMR) were eligible for the metaanalysis [21, 26, 30, 34-36, 38-40].

\section{Qualitative analysis (systematic review)}

\section{Basic study and patient characteristics}

Table 1 summarizes the main characteristics of the 20 included studies. All selected articles have been published in the past decade. Eleven studies (55\%) were performed in Europe, 7 studies (35\%) in Japan and 2 studies (10\%) in Australia. Thirteen studies $(65 \%)$ had a retrospective study design, whilst 7 studies $(35 \%)$ were performed prospectively. Thirteen studies $(65 \%)$ included patients with PMR who underwent [18F]FDG-PET/CT at diagnosis before initiation of glucocorticoid therapy; in 7 studies (35\%), at least part of patients had been treated with glucocorticoid treatment prior to or during the $[18 \mathrm{~F}] \mathrm{FDG}-\mathrm{PET} / \mathrm{CT}$. The reference standard for a diagnosis of PMR consisted of classification criteria in 17 studies (85\%), i.e. the 2012 provisional ACR/EULAR classification criteria for PMR in 7 studies, Chuang's criteria in 5 studies, Bird's criteria in 2 studies, Healey's criteria in 2 studies and a combination of the ACR/EULAR criteria and Bird's criteria in 1 study [4, 41-43]. In 3 studies (15\%), a clinical diagnosis of PMR was used as the reference standard. The included studies were heterogeneous concerning the sex and age of patients.

\section{Technical aspects}

The technical aspects of [18F]FDG-PET/CT in the 20 studies are summarized in Table 2. In 17 studies (85\%), all patients underwent [18F]FDG-PET scanning with low-dose CT. The injected $[18 \mathrm{~F}] \mathrm{FDG}$ activity was quite heterogeneous and included both weight-based and fixed activities. The [18F]FDG incubation time was approximately $60 \mathrm{~min}$ in all studies reporting this technical aspect. The vast majority of scans covered the skull (either from the vertex or skull base) to thigh region whilst some studies also included the knees. Reconstruction algorithms or adherence to EARL was not 
Fig. 1 Study selection flowchart

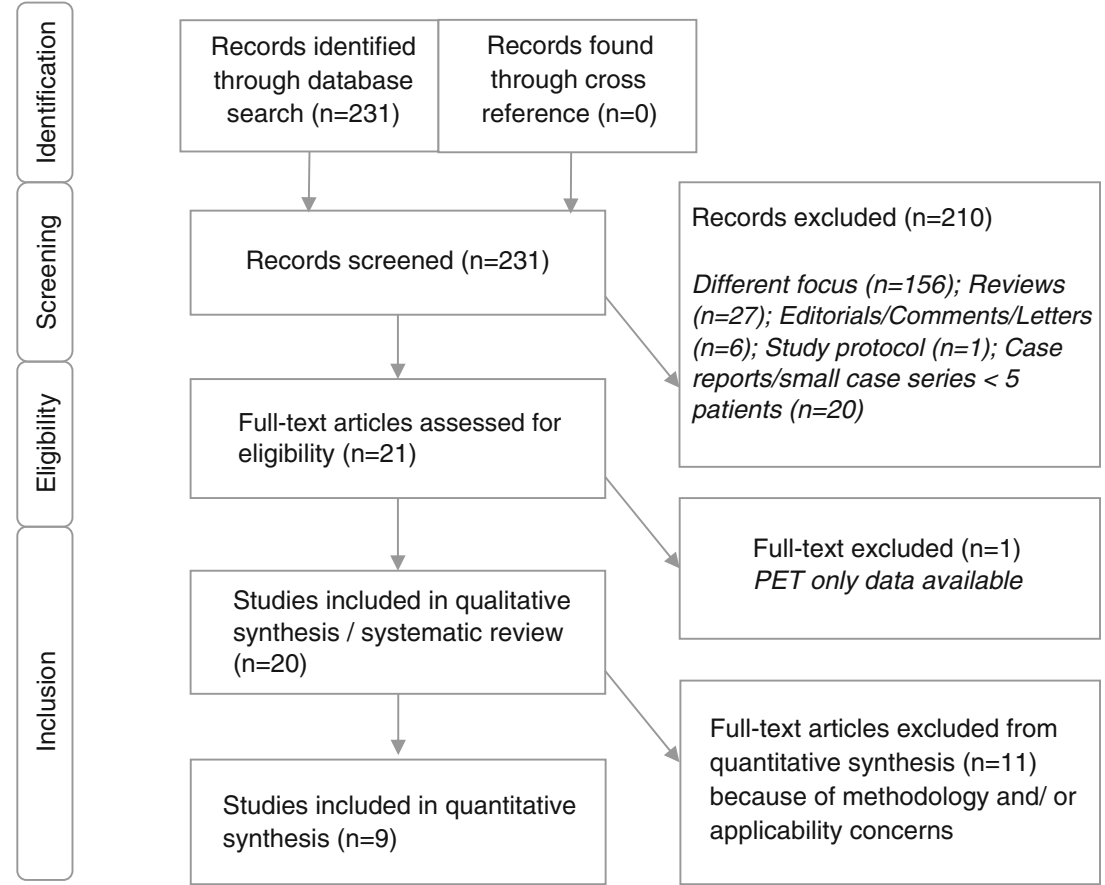

always specified. [18F]FDG-PET/CT image analysis was primarily performed by visual analysis ( 8 studies, $40 \%$ ), semiquantitative analysis using the maximum standardized uptake value $\left(\mathrm{SUV}_{\max }, 3\right.$ studies, $\left.15 \%\right)$ or both of these methods ( $n=9$ studies, $45 \%$ ). In two studies $(10 \%)$, a target-to-liver ratio was used as well. The definition of a positive [18F]FDG uptake was different among the included studies, but the majority of studies used the liver as the reference organ. In 8 studies (40\%), visual uptake equal or higher to the liver was considered positive whilst uptake higher than the liver (either visual or semi-quantitatively) was defined as positive in 5 studies (25\%). Five studies $(25 \%)$ reported a composite [18F]FDG-PET/CT score for PMR, but the anatomic regions included in the score differed per study (Supplementary Table 1).

\section{Main findings of qualitative assessment}

Data regarding the relationship between [18F]FDG-PET/CT and clinical or biochemical findings are provided in Supplementary Table 2. [18F]FDG uptake occurred symmetrically in the shoulder and hip girdles in patients with PMR according to three studies [31, 33, 38]. No convincing relationship was found between [18F]FDG-PET/CT findings and clinical symptoms or inflammatory markers in the blood [21, $23,28,31,37]$. One study evaluated the relationship between the age of onset, response to therapy and [18F]FDG-PET/CT findings [22]. This study demonstrated that young PMR patients $($ age $<60)$ have a relatively low inflammatory burden on [18F]FDG-PET/CT and poor response to glucocorticoid treatment. Two cross-sectional studies compared [18F]FDG$\mathrm{PET} / \mathrm{CT}$ findings between patients with and without concomitant glucocorticoid treatment. Both studies indicated that concomitant glucocorticoid treatment might obscure [18F]FDG-PET/CT findings in patients with PMR [28, 34]. Four studies suggested that [18F]FDG-PET/CT might be useful for monitoring of disease activity in patients treated with glucocorticoids or tocilizumab (antiIL-6 receptor therapy), as indicated by a reduction of $\mathrm{SUV}_{\max }$ values and/or the number of positive sites on the scan after initiation of such therapy [24, 31, 32, 35]. Eight studies evaluated large vessel wall uptake; coexisting large vessel vasculitis was observed in 0 $40 \%$ of patients with PMR $[21,25,29,30,32,33,36$, 39]. In patients initially suspected of PMR, the [18F]FDG-PET/CT scan identified a malignancy in 3$38 \%$ of patients without PMR $[25,26]$.

\section{Quantitative analysis (meta-analysis)}

\section{Studies included in the quantitative analysis}

The 9 studies in the meta-analysis reported [18F]FDG-PET/ CT findings at distinct anatomic sites rather than an overall positive/negative result of the scan. Two studies reported the diagnostic accuracy of a fixed combination of anatomic sites $[21,30]$. Since none of these combinations was reported by more than one study, no meta-analysis was performed for this data. Three unique studies reported the diagnostic accuracy of a composite [18F]FDG-PET/CT score. In case of a potential 


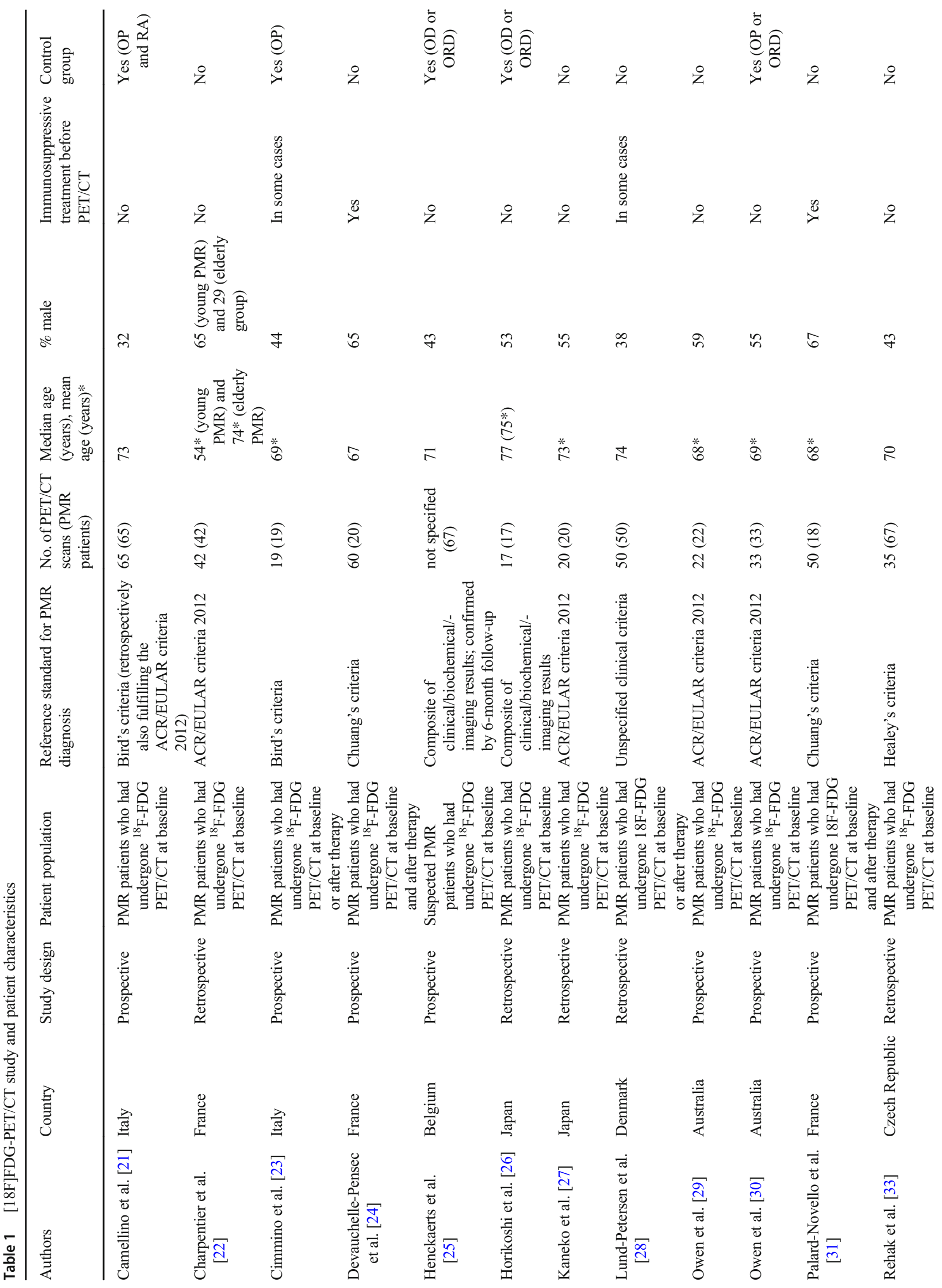




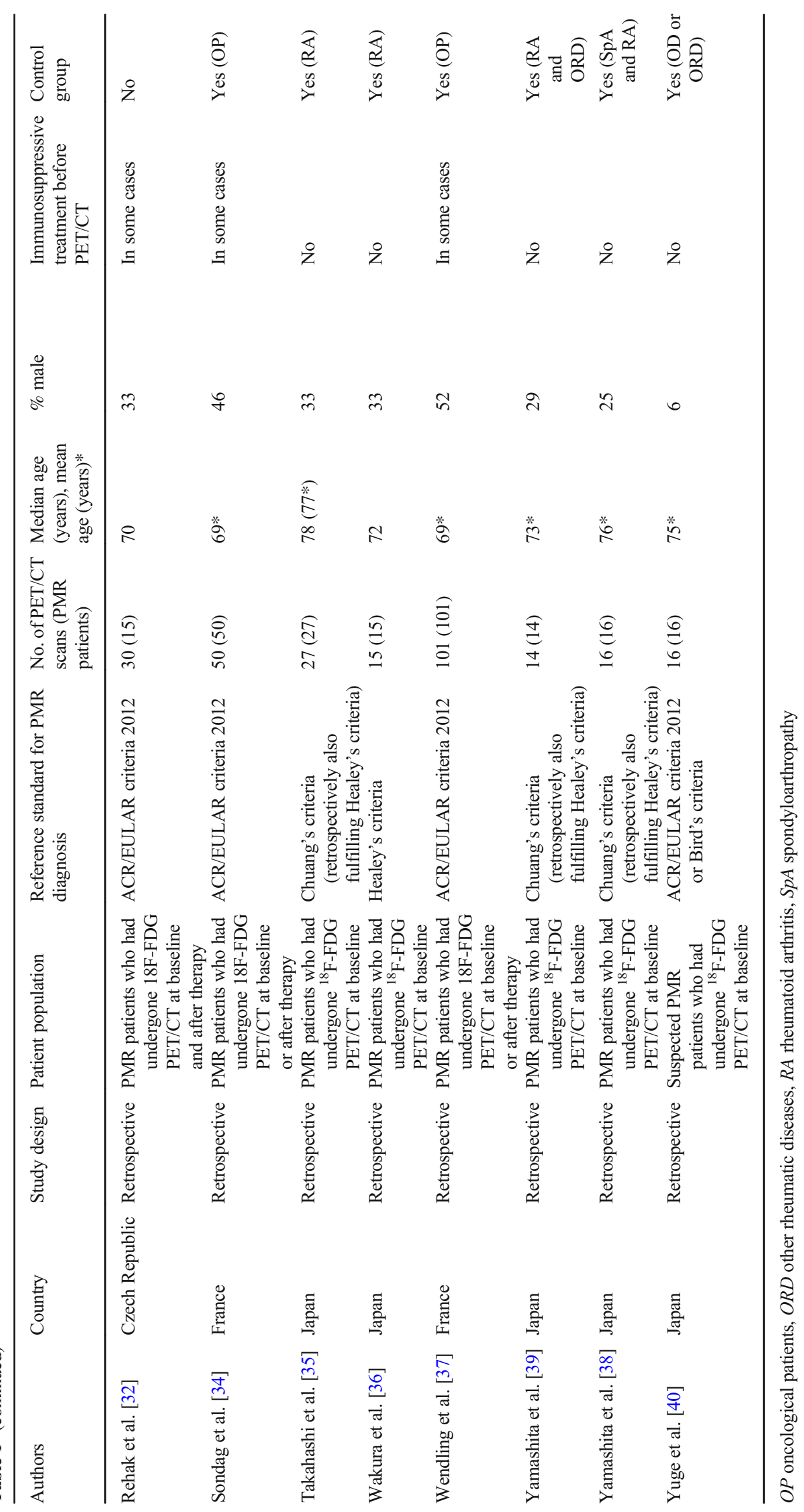




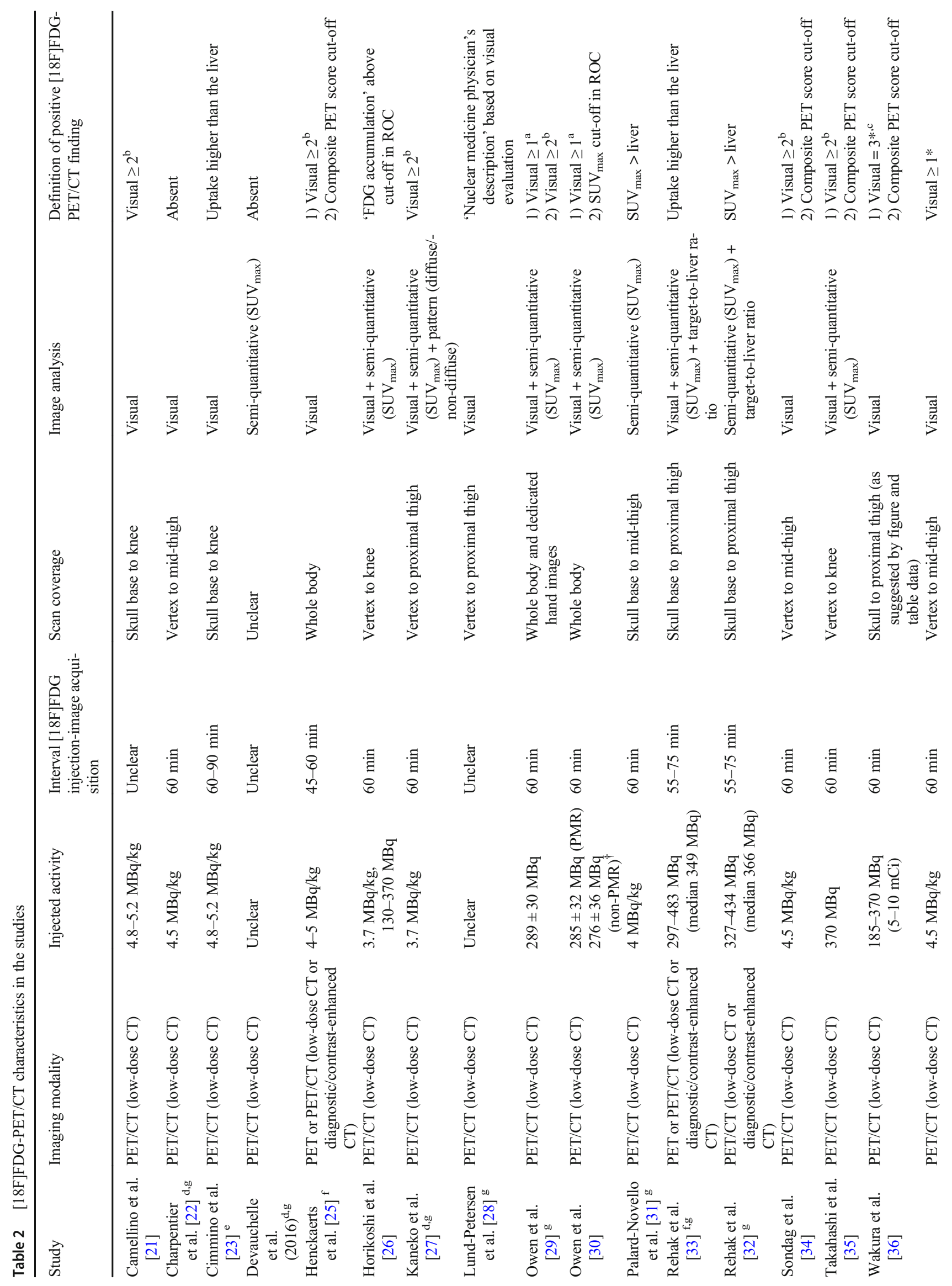




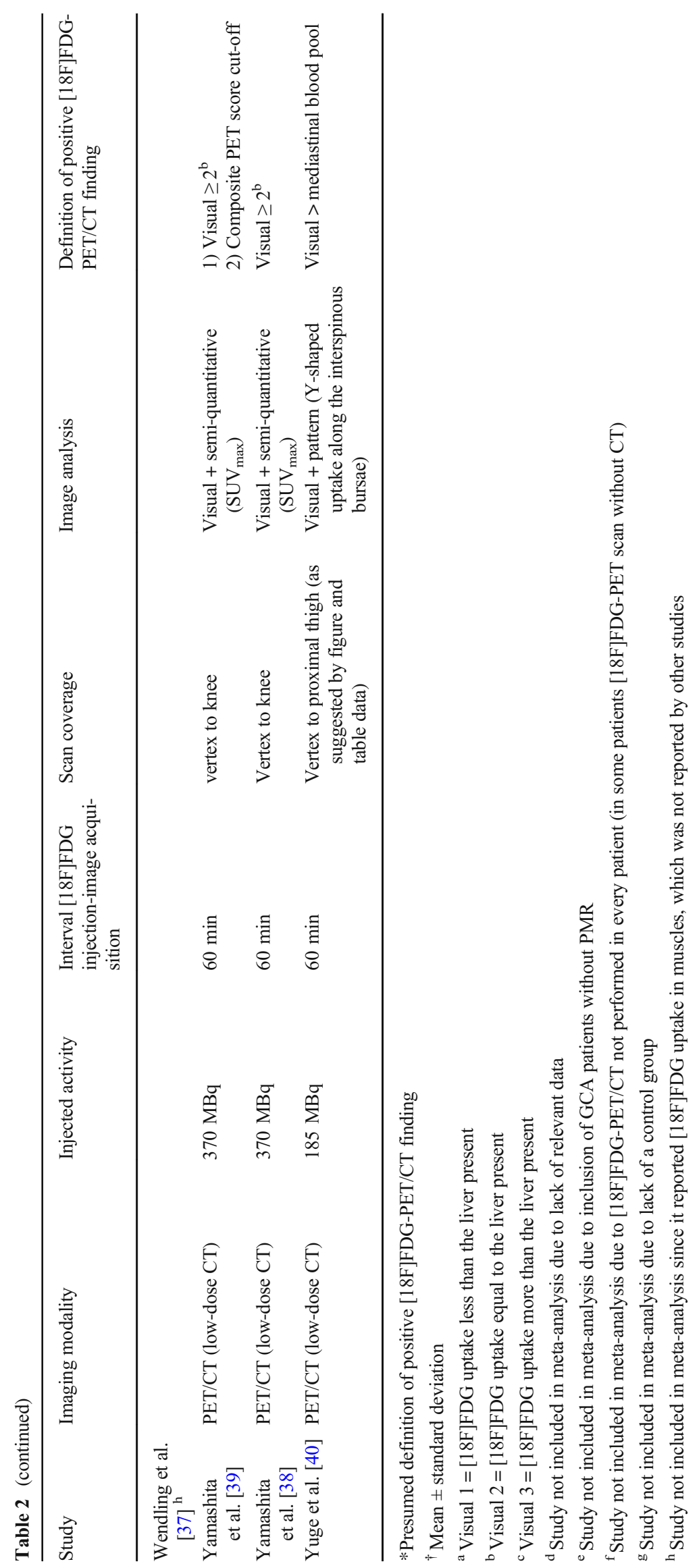




\begin{tabular}{|c|c|c|c|c|c|c|c|}
\hline \multirow[b]{2}{*}{ Study name } & \multicolumn{4}{|c|}{ Risk of bias } & \multicolumn{3}{|c|}{ Applicability concerns } \\
\hline & $\begin{array}{c}\text { Patient } \\
\text { selection }\end{array}$ & Index test & $\begin{array}{c}\text { Reference } \\
\text { standard }\end{array}$ & $\begin{array}{c}\text { Flow and } \\
\text { timing }\end{array}$ & $\begin{array}{c}\text { Patient } \\
\text { selection }\end{array}$ & Index test & $\begin{array}{c}\text { Reference } \\
\text { standard }\end{array}$ \\
\hline Camellino et al. 2014 & : & (;) & (;) & (:) & $?$ & (;) & ;) \\
\hline Horiskoshi et al. 2020 & $?$ & : & : : & ;:) & $?$ & $?$ & (:) \\
\hline Owen et al. 2020 & : & (;) & (:) & (:) & $?$ & (:) & (:) \\
\hline Sondag et al. 2016 & : & (:) & (;) & (:) & $?$ & (:) & (:) \\
\hline Takahashi et al. 2015 & :) & (:) & ;) & (:) & $?$ & (:) & (:) \\
\hline Wakura et al. 2016 & : : & (;) & (;) & (:) & $?$ & ;) & (:) \\
\hline Yamashita et al. 2012 & : & (;) & (:) & (:) & : & (:) & (:) \\
\hline Yamashita et al. 2013 & : & (;) & (;) & (:) & $?$ & (;) & (:) \\
\hline Yuge et al. 2018 & $?$ & ;:) & ;:) & (:) & (:) & (:) & (:) \\
\hline
\end{tabular}

\begin{tabular}{|c|c|c|}
\hline$(;)$ & : & $?$ \\
\hline Low risk & High risk & Unclear \\
\hline
\end{tabular}

Fig. 2 Summary of QUADAS-2 items for the 9 studies included in the meta-analysis

overlap of patients between studies from the same centre, data from only one study were used according to the criteria listed in the "Methods" section (study selection).

\section{Methodological quality of studies in quantitative analysis}

Patient selection was the main source of bias among the 10 studies selected for the meta-analysis (Fig. 2). Two studies did not have a case-control study design and included patients suspected of PMR who underwent a [18F]FDG-PET/CT scan $[26,40]$. Even in the latter two studies, it was unclear whether all patients with suspected PMR, or only a selection of those patients, were scanned.

\section{Diagnostic accuracy of [18F]FDG-PET/CT for PMR}

Table 3 provides an overview of the diagnostic accuracies per anatomic site. The highest pooled sensitivity (>80\%) was observed for positive $[18 \mathrm{~F}] \mathrm{FDG}$ uptake at the ischial tuberosity $(0.85,95 \% \mathrm{CI} 0.62-0.95)$ and greater trochanters $(0.83,95 \%$ CI $0.59-0.95)$, whereas positive [18F]FDG uptake at the interspinous bursae showed the highest specificity (0.81, 95\% CI 0.60-0.93). The LR+ was highest for a positive interspinous bursa on [18F]FDG-PET/CT $(\mathrm{LR}+4.00,95 \% \mathrm{CI}$ 1.84-8.71), followed by [18F]FDG positive hips ( $\mathrm{LR}+2.91$; 95\% CI 2.09-4.05), ischial tuberosities ( $\mathrm{LR}+2.85$; $95 \% \mathrm{CI}$ 1.91-4.25), shoulders (LR+ 2.57; 95\% CI 1.24-5.32) and sternoclavicular joints (LR+ 2.31; 95\% CI 1.33-4.02). The $\mathrm{LR}+$ for the greater trochanter was not statistically significant. All six anatomic sites yielded relevant negative likelihood ratios of less than 0.5 , i.e. ischial tuberosities (LR- 0.21 ; 95\% CI 0.08-0.54), greater trochanters (LR-0.29; 95\% CI 0.13-0.66), interspinous bursae (LR-0.31; 95\% CI $0.21-$ 0.47), shoulders (LR-0.31; 95\% CI 0.19-0.49), hips (LR$0.47 ; 95 \%$ CI $0.31-0.70)$ and sternoclavicular joints (LR0.49 ; 95\% CI $0.29-0.83$ ). Moderate to high heterogeneity was observed for all anatomic sites as shown in the forest plots and HSROC curves (Fig. 3 and Supplementary Fig. 1).

Table 3 Diagnostic accuracy of [18F]FDG-PET/CT findings

\begin{tabular}{|c|c|c|c|c|c|c|}
\hline $\begin{array}{l}\text { Site positive on } \\
{[18 \mathrm{~F}] \mathrm{FDG}-\mathrm{PET} / \mathrm{CT}}\end{array}$ & $\begin{array}{l}\text { No. of patients } \\
\text { (no. of cohorts }{ }^{b} \text { ) }\end{array}$ & $\begin{array}{l}\text { Sensitivity } \\
(95 \% \mathrm{CI})\end{array}$ & $\begin{array}{l}\text { Specificity } \\
(95 \% \text { CI })\end{array}$ & $\begin{array}{l}\text { Diagnostic } \\
\text { OR }(95 \% \mathrm{CI})\end{array}$ & $\mathrm{LR}+(95 \% \mathrm{CI})$ & $\mathrm{LR}-(95 \% \mathrm{CI})$ \\
\hline Hip & $346(5)$ & $63.7(46.3-78.1)$ & $78.1(69.1-85.1)$ & $6.25(3.32-11.79)$ & $2.91(2.09-4.05)$ & $0.47(0.31-0.70)$ \\
\hline Greater trochanter & $428(6)$ & $83.3(59.0-94.5)$ & $56.7(38.3-73.5)$ & $6.54(2.87-14.90)$ & $1.93(1.43-2.59)$ & $0.29(0.13-0.66)$ \\
\hline Interspinous bursa & $546(6)$ & $74.5(59.3-85.4)$ & $81.4(59.6-92.8)$ & $12.76(5.64-28.89)$ & $4.00(1.84-8.71)$ & $0.31(0.21-0.47)$ \\
\hline Ischial tuberosity & $428(6)$ & $85.4(62.3-95.4)$ & $70.1(53.5-82.7)$ & $13.72(5.20-36.18)$ & $2.86(1.91-4.28)$ & $0.21(0.08-0.54)$ \\
\hline Shoulder $^{\mathrm{a}}$ & $406(6)$ & $78.4(65.4-87.5)$ & $69.5(42.5-87.5)$ & $8.30(3.05-22.58)$ & $2.57(1.24-5.32)$ & $0.31(0.19-0.49)$ \\
\hline Sternoclavicular joint & $375(5)$ & $64.4(39.1-83.6)$ & $72.1(48.3-87.8)$ & $4.68(2.06-10.63)$ & $2.31(1.33-4.02)$ & $0.49(0.29-0.83)$ \\
\hline
\end{tabular}

Hierarchical logistic regression modelling was used to determine summary estimates of the sensitivity, specificity, diagnostic odds ratio and likelihood ratios by the bivariate model approach. $95 \%$ CI 95\% confidence interval, $O R$ odds ratio, $L R+$ positive likelihood ratio, $L R-$ negative likelihood ratio

${ }^{a}$ Data either reported as shoulder or glenohumeral joint

${ }^{\mathrm{b}}$ In case of potential data overlap between studies, only data from one study was used according to criteria described in the "Methods" section 


\section{Hip}

Study

Horikoshi et al. 2020

Owen et al. 2020

Sondag et al. 2016

Wakura et al. 2016

Yamashita et al. 2012
TP FP FN TN PET positivity Controls Sensitivity $(95 \% \mathrm{Cl})$ Specificity $(95 \% \mathrm{Cl})$ Sensitivity $(95 \% \mathrm{Cl})$

$\begin{array}{llll}11 & 3 & 6 & 5\end{array}$

$\begin{array}{llll}20 & 26 & 13 & 106\end{array}$

$\begin{array}{llll}19 & 8 & 31 & 45\end{array}$

$\begin{array}{llll}11 & 1 & 4 & 6\end{array}$

$\begin{array}{llll}12 & 6 & 2 & 11\end{array}$

$$
\begin{array}{crr}
\text { Other } & \text { OP or ORD } & 0.65[0.38,0.86] \\
V I S \geq 1 & \text { OP or ORD } & 0.61[0.42,0.77] \\
V I S \geq 2 & \text { OP } & 0.38[0.25,0.53] \\
V I S=3 & \text { RA } & 0.73[0.45,0.92] \\
V I S \geq 2 & \text { RA or ORD } & 0.86[0.57,0.98]
\end{array}
$$

\begin{tabular}{|c|c|c|c|c|c|c|c|c|c|}
\hline Study & TP & FP & FN TN & PET positivity & Controls & Sensitivity (95\% Cl) & Specificity $(95 \% \mathrm{Cl})$ & Sensitivity $(95 \% \mathrm{Cl})$ & Specificity (95\% Cl) \\
\hline Horikoshi et al. 2020 & 11 & 5 & 6 & Other & OP or ORD & $0.65[0.38,0.86]$ & $0.38[0.09,0.76]$ & & \\
\hline Owen et al. 2020 & 33 & 100 & 032 & $V I S \geq 1$ & OP or ORD & $1.00[0.89,1.00]$ & $0.24[0.17,0.32]$ & & $\rightarrow-$ \\
\hline Sondag et al. 2016 & 20 & 8 & $30 \quad 45$ & $V I S \geq 2$ & $\mathrm{OP}$ & $0.40[0.26,0.55]$ & $0.85[0.72,0.93]$ & $\neg-$ & $\longrightarrow$ \\
\hline Wakura et al. 2016 & 14 & 3 & 1 & $V \mid S=3$ & RA & $0.93[0.68,1.00]$ & $0.57[0.18,0.90]$ & & \\
\hline Yamashita et al. 2013 & 13 & 12 & 325 & $V I S \geq 2$ & $\mathrm{RA}$ or SpA & $0.81[0.54,0.96]$ & $0.68[0.50,0.82]$ & & -1- \\
\hline Yuge et al. 2018 & 13 & 21 & 323 & Vis>MBP & ORD & $0.81[0.54,0.96]$ & $0.52[0.37,0.68]$ & & 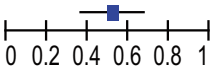 \\
\hline
\end{tabular}

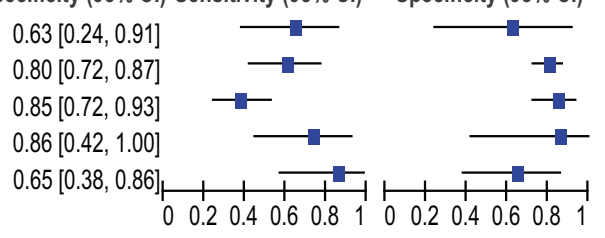

\section{Greater trochanter}

\section{Interspinous bursa}

Study

Camellino et al. 2014

Horikoshi et al. 2020

Owen et al. 2020

Sondag et al. 2016

Yamashita et al. 2013

Yuge et al. 2018
TP FP FN TN

$\begin{array}{llll}31 & 1 & 34 & 74\end{array}$

$\begin{array}{llll}14 & 3 & 3 & 5\end{array}$

$\begin{array}{llll}30 & 23 & 3 & 109\end{array}$

$\begin{array}{llll}29 & 6 & 21 & 47\end{array}$

$\begin{array}{llll}12 & 13 & 4 & 24\end{array}$

$\begin{array}{llll}13 & 23 & 3 & 21\end{array}$

$\begin{array}{cr}\text { PET positivity } & \text { Controls } \\ \text { VIS } \geq 2 & \text { OP or } R A \\ \text { Other } & \text { OP or ORD } \\ \text { VIS } \geq 1 & \text { OP or ORD } \\ \text { VIS } \geq 2 & \text { OP } \\ \text { VIS } \geq 2 & \text { RA or } S p A \\ \text { Vis }>\text { MBP } & \text { ORD }\end{array}$

\begin{tabular}{|c|c|c|c|c|c|c|c|c|c|c|}
\hline Study & TP & FP & $\mathrm{FN}$ & TN & PET positivity & Controls & Sensitivity $(95 \% \mathrm{Cl})$ & Specificity $(95 \% \mathrm{Cl})$ & Sensitivity $(95 \% \mathrm{Cl})$ & Specificity $(95 \% \mathrm{Cl})$ \\
\hline Horikoshi et al. 2020 & 10 & 4 & 7 & 4 & Other & OP or ORD & $0.59[0.33,0.82]$ & $0.50[0.16,0.84]$ & & \\
\hline Owen et al. 2020 & 33 & 77 & 0 & 55 & $V I S \geq 1$ & OP or ORD & $1.00[0.89,1.00]$ & $0.42[0.33,0.51]$ & $\rightarrow$ & - \\
\hline Sondag et al. 2016 & 28 & 4 & 22 & 49 & $V I S \geq 2$ & OP & $0.56[0.41,0.70]$ & $0.92[0.82,0.98]$ & & - \\
\hline Wakura et al. 2016 & 14 & 1 & 1 & 6 & $V I S=3$ & RA & $0.93[0.68,1.00]$ & $0.86[0.42,1.00]$ & & \\
\hline Yamashita et al. 2013 & 15 & 15 & 1 & 22 & $V I S \geq 2$ & RA or SpA & $0.94[0.70,1.00]$ & $0.59[0.42,0.75]$ & & - \\
\hline Yuge et al. 2018 & 11 & 12 & 5 & 32 & $V i s>M B P$ & ORD & $0.69[0.41,0.89]$ & $0.73[0.57,0.85]_{\ldots}$ & $\Rightarrow \frac{}{1}$ & +5 \\
\hline
\end{tabular}

Sensitivity $(95 \% \mathrm{Cl})$ Specil

$0.48[0.35,0.60]$

$0.82[0.57,0.96]$

$0.91[0.76,0.98]$

$0.58[0.43,0.72]$

$0.75[0.48,0.93]$

$0.81[0.54,0.96]$

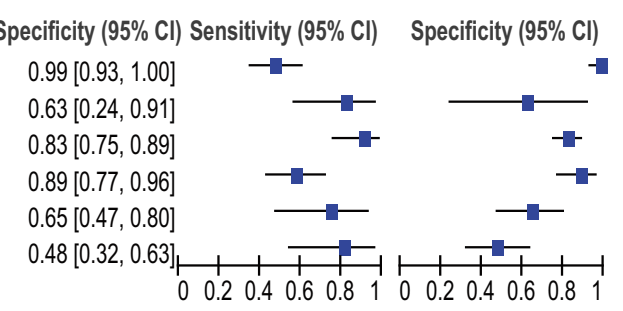

\section{Ischial tuberosity}

\section{Shoulder}

Study

Horikoshi et al. 2020

Owen et al. 2020

Sondag et al. 2016

Wakura et al. 2016

Yamashita et al. 2012

Yuge et al. 2018
TP FP FN TN PETPO

$\begin{array}{llll}15 & 2 & 2 & 6\end{array}$

$\begin{array}{llll}24 & 6 & 9 & 126\end{array}$

$\begin{array}{llll}29 & 8 & 21 & 45\end{array}$

$\begin{array}{llll}12 & 4 & 3 & 3\end{array}$

$\begin{array}{llll}12 & 12 & 2 & 5\end{array}$

$\begin{array}{llll}14 & 22 & 2 & 22\end{array}$
PET positivity Controls Se

Other OP or ORD

VIS $\geq 1$ OP or ORD

$V I S \geq 2 \quad O P$

$V I S=3 \quad$ RA

$V I S \geq 2$ RA or ORD

Vis>MBP ORD
Sensitivity (95\% Cl) Specificity (95\% Cl) Sensitivity (95\% C

$0.88[0.64,0.99]$

$0.73[0.54,0.87]$

$0.58[0.43,0.72]$

\begin{tabular}{|c|c|c|c|c|c|c|c|c|c|c|}
\hline Study & TP & FP & $\mathrm{FN}$ & TN & PET positivity & Controls & Sensitivity $(95 \% \mathrm{Cl})$ & Specificity $(95 \% \mathrm{Cl})$ & Sensitivity (95\% Cl) & Specificity $(95 \% \mathrm{Cl})$ \\
\hline Horikoshi et al. 2020 & 9 & 5 & 8 & 3 & Other & OP or ORD & $0.53[0.28,0.77]$ & $0.38[0.09,0.76]$ & & $\square$ \\
\hline Owen et al. 2020 & 29 & 75 & 4 & 57 & $V I S \geq 1$ & OP or ORD & $0.88[0.72,0.97]$ & $0.43[0.35,0.52]$ & 一- & - \\
\hline Sondag et al. 2016 & 12 & 3 & 38 & 50 & $V I S \geq 2$ & $\mathrm{OP}$ & $0.24[0.13,0.38]$ & $0.94[0.84,0.99]$ & $\rightarrow-$ & $\rightarrow$ \\
\hline Wakura et al. 2016 & 9 & 2 & 6 & 5 & $V I S=3$ & RA & $0.60[0.32,0.84]$ & $0.71[0.29,0.96]$ & & $-1-$ \\
\hline Yuge et al. 2018 & 14 & 11 & 2 & 33 & $V i s>M B P$ & ORD & $0.88[0.62,0.98]$ & $0.75[0.60,0.87]_{\mathrm{t}}$ & 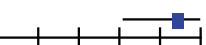 & -5 \\
\hline
\end{tabular}

$0.80[0.52,0.96]$

$0.86[0.57,0.98]$

$0.88[0.62,0.98]$

$0.85[0.72,0.93]$
$0.75[0.35,0.97]$

$0.95[0.90,0.98]$

$0.43[0.10,0.82]$

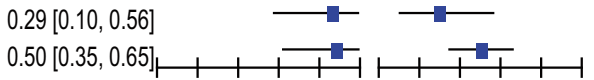

\section{Sternoclavicular joint}

Fig. 3 Forest plots showing the sensitivity and specificity of [18F]FDGPET/CT for PMR. Data are shown for the anatomic sites reported by at least 4 unique studies. VIS visual uptake, OP oncologic patients, ORD patients with other rheumatic disease, RA patients with rheumatoid arthritis, SpA patients with spondyloarthritis, MBP mediastinal blood pool 
Diagnostic accuracy data regarding sites reported by less than 4 studies are provided in Supplementary Table 3. Three studies reported on a composite [18F]FDG-PET/CT score with a pooled LR+ of 3.91 (95\% CI 2.42-6.32) and LR- of 0.19 (95\% CI $0.10-0.36)$ at the optimal cut-off points.

\section{Discussion}

\section{Main findings}

This systematic review and meta-analysis summarizes current evidence on the diagnostic value of [18F]FDG-PET/CT for PMR. Estimates of the LRs indicate that shoulders, sternoclavicular joints, interspinous bursae, ischial tuberosities, hips and greater trochanters are important anatomic sites to evaluate in patients with suspected PMR. Concomitant use of glucocorticoid treatment may affect the sensitivity of the [18F]FDG-PET/CT for diagnosing PMR. A limited number of studies suggest that [18F]FDG-PET/CT might be useful for the monitoring of disease activity in patients with PMR. Moderate to high heterogeneity was observed across studies not only due to selection bias, but also due to differences in scanning procedures and interpretation.

Since various articular and extra-articular sites throughout the body can be involved in PMR, a whole-body evaluation of inflammatory activity by [18F]FDG-PET/CT offers significant advantages over localized MRI or ultrasonography [12]. Ultrasonography (sensitivity $66 \%$, specificity $81 \%$ ) is currently recommended as a diagnostic imaging modality for suspected PMR according to the 2012 provisional ACR/ EULAR classification criteria for PMR [4]. Our study indicates that [18F]FDG-PET/CT findings at various individual anatomic sites provide comparable sensitivity and specificity for a diagnosis of PMR. Moreover, composite [18F]FDG$\mathrm{PET} / \mathrm{CT}$ scores provided a pooled sensitivity of $85 \%$ and a specificity of $80 \%$. Given its higher sensitivity and similar specificity compared to ultrasound, [18F]FDG-PET/CT is a valuable diagnostic tool, especially in patients with clinically suspected PMR and negative ultrasound scan. More recently, combined MRI of shoulders and hips has been shown to allow for a more accurate assessment of joint and peri-articular inflammation compared to ultrasound [10]. Mackie et al. have reported on a typical 'extracapsular pattern' on multiple joint MRI, yielding a specificity of $94 \%$ and a sensitivity of $64 \%$ for diagnosing PMR [7]. Unlike ultrasonography and MRI, [18F]FDG-PET/CT is inherently a whole-body imaging modality and allows evaluating other disorders such as associated large vessel vasculitis or malignancies. Such conditions were indeed identified by [18F]FDG-PET/CT in some of the studies included in our systematic review. Overall, there is accumulating evidence pointing towards a valuable role for $[18 \mathrm{~F}]-$
FDG-PET/CT in the diagnostic work-up of patients with suspected PMR.

Important anatomic sites in the evaluation of suspected PMR by [18F]FDG-PET/CT encompassed the articular and extra-articular structures of the shoulder and pelvic girdle, as well as the spinal column. Although insufficient data precluded evaluation of knee [18F]FDG uptake in the current metaanalysis, it has been suggested that knees can be affected in PMR and should also be evaluated if possible [12, 23, 29, 30]. It would be interesting to know the diagnostic accuracy of fixed combinations of distinct anatomic sites, for instance involvement of shoulders and ischial tuberosities on [18F]FDG$\mathrm{PET} / \mathrm{CT}$. This combination provided a sensitivity of $94 \%$ and a specificity of $92 \%$ for PMR in one study [30]. However, data for such combinations were too scarce to include in the current meta-analysis. Nevertheless, three unique studies allowed evaluating the diagnostic accuracy of a composite [18F]FDG-PET/CT score for PMR [34-36]. Although the scoring systems were very different, rather homogeneous diagnostic accuracy data were obtained with a pooled sensitivity and specificity of $85 \%$ and $80 \%$, respectively. The study by Henckaerts et al., which was omitted from the meta-analysis due to inclusion of patients with [18F]FDG-PET scans, reported a similar diagnostic accuracy for another composite [18F]FDG-PET/CT score [25]. Future studies should determine which composite [18F]FDG-PET/CT score is preferred.

Recently, another meta-analysis by Kim et al. evaluated the diagnostic performance of [18F]FDG-PET/CT for PMR [44]. The latter included two studies that were excluded from our meta-analysis (one due to inclusion of PET scans without CT and the other one because of reporting muscle metabolic activity), whilst 4 additional studies have been included in our meta-analysis [21, 25, 26, 37-39]. Our risk of bias assessment concerning patient selection differed substantially. Most studies in both meta-analyses were case-control studies in which the control subjects were not necessarily suspected of having PMR and were therefore considered to be at high risk for selection bias in our study. The meta-analysis by Kim et al. suggested a pooled sensitivity of $76 \%$ and a specificity of $76 \%$ of overall [18F]FDG-PET/CT positivity for a diagnosis of PMR, although a precise definition for overall [18F]FDG$\mathrm{PET} / \mathrm{CT}$ positivity was not provided. In contrast to the metaanalysis by Kim et al., our study provides more detailed data including the evaluation of composite [18F]FDG-PET/CT scores and diagnostic accuracy of [18F]FDG-PET/CT findings at distinct anatomic sites, as well as an extensive qualitative assessment.

Several factors might have contributed to the betweenstudy heterogeneity observed in the forest plots and HSROC curves. First, differences in methodological aspects of the [18F]FDG-PET/CT scan (e.g. administered activity, scan systems, reconstruction algorithms) could lead to such heterogeneity. Moreover, variation in scoring systems was observed 
across the included studies. All studies included in the metaanalysis applied a visual uptake scoring system, whilst half of these studies also applied a semi-quantitative parameter (i.e. $\left.\mathrm{SUV}_{\max }\right)$. The visual grading system mainly used the liver activity as the reference background, but the definition of FDG positivity on a visual scale as well as the optimal SUV cut-off value differed substantially between the studies. This highlights the need for a standardized scoring system for PMR activity on [18F]FDG-PET/CT in addition to standardization of the scanning protocol itself. Importantly, procedural recommendations for [18F]FDG-PET/CT imaging in PMR have recently been reported [12]. The between-study heterogeneity could also be explained by differences in patient characteristics in the included studies. For instance, most studies were case-controlled studies and the selection of the control cohort (e.g. patients with cancer, or rheumatoid arthritis) might have heavily influenced the observed diagnostic accuracy of [18F]FDG-PET/CT.

\section{Limitations}

We do acknowledge further limitations of our study. The number of patients included in the meta-analysis was relatively small. Due to exclusion of non-English reports or conference papers, relevant data may have been omitted. We did not seek to obtain unpublished data via contacting of authors. Various types of bias were present in our study. Most studies had a case-control design. The selection of a control group without symptoms suggestive of PMR (e.g. oncologic patients) might lead to overestimation of the diagnostic accuracy of [18F]FDG-PET/CT for PMR. Additional selection bias may have resulted from the retrospective nature of the majority of the studies. For instance, the decision to perform a [18F]FDG-PET/CT might be based on the clinical suspicion for a malignancy or concomitant large vessel vasculitis. In a minority of studies, some patients had already received glucocorticoid treatment prior to the [18F]FDG-PET/CT, which might have led to underestimation of the diagnostic accuracy. Our systematic review was primarily focused on PMR in the absence of giant cell arteritis, although concomitant vasculitis was observed in part of the included studies. Finally, publication bias is a concern inherent to all meta-analyses. Whilst these factors need to be taken into account, the current study provides the most comprehensive overview of the diagnostic value of [18F]FDG-PET/CT for PMR to date.

\section{Conclusion}

[18F]FDG-PET/CT may be a valuable diagnostic tool in the work-up of patients with suspected PMR, and this study provides insight into specific anatomic sites on [18F]FDG-PET/ $\mathrm{CT}$ that are informative for a diagnosis of PMR. A composite
[18F]FDG-PET/CT score might also be of interest, but agreement on the preferred anatomic sites in such composite score is awaited. Depending on the clinical probability of PMR, [18F]FDG-PET/CT may help to rule in or rule out the diagnosis. Furthermore, [18F]FDG-PET/CT aids in the detection of other serious conditions in part of patients. Further studies are needed to more precisely estimate the diagnostic accuracy of [18F]FDG-PET/CT for PMR. Such studies should ideally have a prospective study design, include all consecutive patients with suspected PMR and adhere to reported procedural recommendations and interpretation criteria for [18F]FDG$\mathrm{PET} / \mathrm{CT}$ in PMR.

Supplementary Information The online version contains supplementary material available at https://doi.org/10.1007/s00259-020-05162-6.

Funding Open access funding provided by University Medical Center Groningen (UMCG).

\section{Compliance with ethical standards}

Conflict of interest Dr. van der Geest has received a speaker fee from Roche paid to the UMCG. Dr. Brouwer has received consultancy and speaker fees from Roche paid to the UMCG. The other authors have no disclosures.

Ethics approval Not required since no human participants or animals were recruited for the current study.

Open Access This article is licensed under a Creative Commons Attribution 4.0 International License, which permits use, sharing, adaptation, distribution and reproduction in any medium or format, as long as you give appropriate credit to the original author(s) and the source, provide a link to the Creative Commons licence, and indicate if changes were made. The images or other third party material in this article are included in the article's Creative Commons licence, unless indicated otherwise in a credit line to the material. If material is not included in the article's Creative Commons licence and your intended use is not permitted by statutory regulation or exceeds the permitted use, you will need to obtain permission directly from the copyright holder. To view a copy of this licence, visit http://creativecommons.org/licenses/by/4.0/.

\section{References}

1. Crowson CS, Matteson EL, Myasoedova E, Michet CJ, Ernste FC, Warrington KJ, et al. The lifetime risk of adult-onset rheumatoid arthritis and other inflammatory autoimmune rheumatic diseases. Arthritis Rheum. 2011;63:633-9.

2. González-Gay MA, Matteson EL, Castañeda S. Polymyalgia rheumatica. Lancet. 2017;390:1700-12.

3. van der Geest KSM, Abdulahad WH, Rutgers A, Horst G, Bijzet J, Arends S, et al. Serum markers associated with disease activity in giant cell arteritis and polymyalgia rheumatica. Rheumatology. 2015;54:1397-402.

4. Dasgupta B, Cimmino MA, Maradit-Kremers H, Schmidt WA, Schirmer M, Salvarani C, et al. 2012 provisional classification criteria for polymyalgia rheumatica: a European League Against 
Rheumatism/American College of Rheumatology collaborative initiative. Ann Rheum Dis. 2012;71:484-92.

5. Macchioni P, Catanoso MG, Pipitone N, Boiardi L, Salvarani C. Longitudinal examination with shoulder ultrasound of patients with polymyalgia rheumatica. Rheumatology. 2009;48:1566-9.

6. Suzuki T, Yoshida R, Hidaka Y, Seri Y. Proliferative synovitis of the shoulder bursae is a key feature for discriminating elderly onset rheumatoid arthritis mimicking polymyalgia rheumatica from polymyalgia rheumatica. Clin Med Insights Arthritis Musculoskelet Disord. 2017;10:117954411774585.

7. Mackie SL, Pease CT, Fukuba E, Harris E, Emery P, Hodgson R, et al. Whole-body MRI of patients with polymyalgia rheumatica identifies a distinct subset with complete patient-reported response to glucocorticoids. Ann Rheum Dis. 2015;74:2188-92.

8. Laporte J-P, Garrigues F, Huwart A, Jousse-Joulin S, Marhadour T, Guellec D, et al. Localized myofascial inflammation revealed by magnetic resonance imaging in recent-onset polymyalgia rheumatica and effect of tocilizumab therapy. J Rheumatol. 2019;46:1619-26.

9. Fruth B, Buehring B, Baraliakos X, Braun J. Use of contrastenhanced magnetic resonance imaging of the pelvis to describe changes at different anatomic sites which are potentially specific for polymyalgia rheumatica. Clin Exp Rheumatol. 2018;36:S8695 .

10. Huwart A, Garrigues F, Jousse-Joulin S, Marhadour T, Guellec D, Cornec $\mathrm{D}$, et al. Ultrasonography and magnetic resonance imaging changes in patients with polymyalgia rheumatica treated by tocilizumab. Arthritis Res Ther. 2018;20:11.

11. Glaudemans AWJM, de Vries EFJ, Galli F, Dierckx RAJO, Slart RHJA, Signore A. The use of (18)F-FDG-PET/CT for diagnosis and treatment monitoring of inflammatory and infectious diseases. Clin Dev Immunol. 2013;2013:1-14.

12. Slart RHJA. FDG-PET/CT(A) imaging in large vessel vasculitis and polymyalgia rheumatica: joint procedural recommendation of the EANM, SNMMI, and the PET Interest Group (PIG), and endorsed by the ASNC. Eur J Nucl Med Mol Imaging. 2018;45: 1250-69.

13. Fu Y, Maianu L, Melbert B, Garvey W. Facilitative glucose transporter gene expression in human lymphocytes, monocytes, and macrophages: a role for GLUT isoforms 1, 3, and 5 in the immune response and foam cell formation. Blood Cells Mol Dis. 2004;32: 182-90.

14. Yamada S, Kubota K, Kubota R, Ido T, Tamahashi N. High accumulation of fluorine-18-fluorodeoxyglucose in turpentine-induced inflammatory tissue. J Nucl Med. 1995;36:1301-6.

15. Kubota K, Yamashita H, Mimori A. Clinical value of FDG-PET/ $\mathrm{CT}$ for the evaluation of rheumatic diseases: rheumatoid arthritis, polymyalgia rheumatica, and relapsing polychondritis. Semin Nucl Med. 2017;47:408-24.

16. Dejaco C, Ramiro S, Duftner C, Besson FL, Bley TA, Blockmans $\mathrm{D}$, et al. EULAR recommendations for the use of imaging in large vessel vasculitis in clinical practice. Ann Rheum Dis. 2018;77:63643.

17. McInnes MDF, Moher D, Thombs BD, McGrath TA, Bossuyt PM, Clifford T, et al. Preferred reporting items for a systematic review and meta-analysis of diagnostic test accuracy studies. JAMA. 2018;319:388.

18. Whiting PF, Rutjes A, Westwood M. QUADAS-2: a revised tool for the quality assessment of diagnostic accuracy studies. Ann Intern Med. 2011;155:529-236.

19. Blockmans D, Maes A, Stroobants S, Nuyts J, Bormans G, Knockaert D, et al. New arguments for a vasculitic nature of polymyalgia rheumatica using positron emission tomography. Rheumatology. 1999.

20. Blockmans D, De Ceuninck L, Vanderschueren S, Knockaert D, Mortelmans L, Bobbaers H. Repetitive 18F-fluorodeoxyglucose positron emission tomography in giant cell arteritis: a prospective study of 35 patients. Arthritis Care Res. 2006;55:131-7.

21. Camellino D, Paparo F, Morbelli S, Cutolo M, Sambuceti G, Cimmino MA. Interspinous bursitis is common in polymyalgia rheumatica, but is not associated with spinal pain. Arthritis Res Ther. BioMed Central Ltd.; 2014;16.

22. Charpentier A, Verhoeven F, Sondag M, Guillot X, Prati C, Wendling D. Therapeutic response to prednisone in relation to age in polymyalgia rheumatica: a comparison study. Clin Rheumatol. Springer London. 2018;37:819-23.

23. Cimmino MA, Camellino D, Paparo F, Morbelli S, Massollo M, Cutolo M, et al. High frequency of capsular knee involvement in polymyalgia rheumatica/giant cell arteritis patients studied by positron emission tomography. Rheumatol (United Kingdom). Oxford University Press. 2013;52:1865-72.

24. Devauchelle-Pensec V, Berthelot JM, Cornec D, Renaudineau Y, Marhadour T, Jousse-Joulin S, et al. Efficacy of first-line tocilizumab therapy in early polymyalgia rheumatica: a prospective longitudinal study. Ann Rheum DisBMJ Publishing Group. 2016;75:1506-10.

25. Henckaerts L, Gheysens O, Vanderschueren S, Goffin K, Blockmans D. Use of $18 \mathrm{f}$-fluorodeoxyglucose positron emission tomography in the diagnosis of polymyalgia rheumatica - a prospective study of 99 patients. Rheumatol (United Kingdom). Oxford University Press. 2018;57:1908-16.

26. Horikoshi H, Nakanishi T, Tamura K, Kimura F, Itoh K. 18Ffluorodeoxyglucose-positron emission tomography/computed tomography for the diagnosis of polymyalgia-like illnesses: a retrospective study. BMC Rheumatol. BioMed Central Ltd.; 2020;4.

27. Kaneko K, Suematsu E, Miyamura T, Ishioka H. Differences of articular and extra-articular involvement in polymyalgia rheumatica: a comparison by whole-body FDG-PET/CT. Mod Rheumatol Taylor and Francis Ltd. 2020;30:358-64.

28. Lund-Petersen A, Voss A, Laustrup H. PET-CT findings in patients with polymyalgia rheumatica without symptoms of cranial ischaemia. Dan Med J. 2017;64:A5410.

29. Owen CE, Poon AMT, Lee ST, Yap LP, Zwar RB, McMenamin $\mathrm{CM}$, et al. Fusion of positron emission tomography/computed tomography with magnetic resonance imaging reveals hamstring peritendonitis in polymyalgia rheumatica. Rheumatol (United Kingdom). Oxford University Press. 2018;57:345-53.

30. Owen CE, Poon AMT, Yang V, McMaster C, Lee ST, Liew DFL, et al. Abnormalities at three musculoskeletal sites on whole-body positron emission tomography/computed tomography can diagnose polymyalgia rheumatica with high sensitivity and specificity. Eur J Nucl Med Mol Imaging Springer. 2020;47:2461-8.

31. Palard-Novello X, Querellou S, Gouillou M, Saraux A, Marhadour T, Garrigues F, et al. Value of 18F-FDG PET/CT for therapeutic assessment of patients with polymyalgia rheumatica receiving tocilizumab as first-line treatment. Eur J Nucl Med Mol Imaging. Springer Berlin. 2016;43:773-9.

32. Rehak Z, Sprlakova-Pukova A, Bortlicek Z, Fojtik Z, Kazda T, Joukal $\mathrm{M}$, et al. PET/CT imaging in polymyalgia rheumatica: praepubic 18F-FDG uptake correlates with pectineus and adductor longus muscles enthesitis and with tenosynovitis. Radiol Oncol Association of Radiology and Oncology. 2017;51:8-14.

33. Rehak Z, Vasina J, Nemec P, Fojtik Z, Koukalova R, Bortlicek Z, et al. Various forms of $18 \mathrm{~F}$-FDG PET and PET/CT findings in patients with polymyalgia rheumatica. Biomed Pap PALACKY UNIV. 2015;159:629-36.

34. Sondag M, Guillot X, Verhoeven F, Blagosklonov O, Prati C, Boulahdour $\mathrm{H}$, et al. Utility of $18 \mathrm{~F}$-fluoro-dexoxyglucose positron emission tomography for the diagnosis of polymyalgia rheumatica: a controlled study. Rheumatol (United Kingdom). Oxford University Press. 2016;55:1452-7. 
35. Takahashi H, Yamashita H, Kubota K, Miyata Y, Okasaki M, Morooka M, et al. Differences in fluorodeoxyglucose positron emission tomography/computed tomography findings between elderly onset rheumatoid arthritis and polymyalgia rheumatica. Mod Rheumatol Informa Healthcare. 2015;25:546-51.

36. Wakura D, Kotani T, Takeuchi T, Komori T, Yoshida S, Makino S, et al. Differentiation between polymyalgia rheumatica (PMR) and elderly-onset rheumatoid arthritis using 18F-fluorodeoxyglucose positron emission tomography/computed tomography: is enthesitis a new pathological lesion in PMR? PLoS One Public Library of Science. 2016;11.

37. Wendling D, Sondag M, Giraud N, Chouk M, Boulahdour H, Prati $\mathrm{C}$, et al. Muscle involvement on 18F-FDG PET-CT in polymyalgia rheumatica. a controlled retrospective study of 101 patients. Jt Bone Spine. Elsevier Masson SAS2020;87:225-8.

38. Yamashita H, Kubota K, Takahashi Y, Minamimoto R, Morooka $\mathrm{M}$, Kaneko H, et al. Similarities and differences in fluorodeoxyglucose positron emission tomography/computed tomography findings in spondyloarthropathy, polymyalgia rheumatica and rheumatoid arthritis. Jt Bone Spine. 2013;80:171-7.

39. Yamashita H, Kubota K, Takahashi Y, Minaminoto R, Morooka M, Ito $\mathrm{K}$, et al. Whole-body fluorodeoxyglucose positron emission tomography/computed tomography in patients with active polymyalgia rheumatica: evidence for distinctive bursitis and large-vessel vasculitis. Mod Rheumatol. 2012;22:705-11.

40. Yuge S, Nakatani K, Yoshino K, Koyama T. Diagnosing polymyalgia rheumatica on 18F-FDG PET/CT: typical uptake patterns. Ann Nucl Med Springer Tokyo. 2018;32:573-7.

41. Bird HA, Esselinckx W, Dixon SAJ, Mowat AG, Wood PH. An evaluation of criteria for polymyalgia rheumatica. Ann Rheum Dis. 1979:434-9.

42. Chuang TY, Hunder GG, Ilstrup DM, Kurland LT. Polymyalgia rheumatica. a 10-year epidemiologic and clinical study. Ann Intern Med. 1982;672-80.

43. Healey LA. Long-term follow-up of polymyalgia rheumatica: evidence for synovitis. Semin Arthritis Rheum. 1984:322-8.

44. Kim K, Kim S-J. Diagnostic performance of F-18 fluorodeoxyglucose PET/computed tomography for diagnosis of polymyalgia rheumatica: a meta-analysis. Nucl Med Commun. 2020;41:1313-21.

Publisher's note Springer Nature remains neutral with regard to jurisdictional claims in published maps and institutional affiliations. 